The strength of the 'sedimentary' layer may be of the order of $10^{9}$ or $10^{10}$ dynes per centimetre and the viscosity of the immediate interior according to one estimate is of the order of $10^{22}$ poises.

During such a period of yielding the sedimentary rocks may become folded and faulted, the process being accompanied by earthquakes with foci in the 'sedimentary' layer, seldom in the 'granitic' layer, and less frequently in the 'intermediate' layer. The movement may be started by any load of sufficient magnitude applied in one place such as the accumulation of ice during an ice age. Scotland and Fennoscandia are now rising after their Pleistocene ice has been melted away, while south-east England is falling at the rate of about a foot per century. This twisting may have set up strains in the upper layers, causing the North Sea earthquakes. Many of the Norwegian earthquakes are superficial.

Experiments made in the Geophysical Laboratory at Washington tend to confirm that rocks behave differently under forces gradually applied from what they do under forces applied quickly. The material of the earth's crust is continually tending towards hydrodynamical equilibrium. To this equilibrium the crust is continuously approaching with the passage of time, but the equilibrium is never absolutely attained owing to the resistive strength of the materials. This is Dutton's conception of isostasy. The work of Hayford, Heiskanen, the Indian Ordnance and Geological Surveys and others tends to show that although Hayford's numerical conception of isostasy has distinct computational advantages in geodesy, involving as it does gravity anomalies, yet on the available evidence it is effective over about one per cent of the earth's surface and ineffective over another 0.75 per cent. This is the extent of the earth's surface so far surveyed in this manner. Tests are now being made with seismographs in the neighbourhood of the Grand Coulee and Shasta Dams in the United States to see whether or not a small area suddenly loaded gives the necessary conditions for small earthquakes to occur. Numerous small earthquakes occur near Boulder Dam, but the pre-dam seismic history is not known.

Earthquakes with epicentres in areas subject to recent geological tectonic activity tend to have their foci in the 'sedimentary' layer, whereas foci in the 'intermediate' layer and down to $300 \mathrm{~km}$. depths show some correlation with zones of Tertiary mountain building. There is still something to be said for the contracting earth theory though perhaps such forces as the Polfluchtkraft require more careful comment. Much seems to depend on the structure of, say, the upper $100 \mathrm{~km}$. of the earth. The bed of the Pacific Ocean appears to be the only region in the world where the 'intermediate' and 'granitic' layers are absent. In the Pacific region the sima comes right to the surface, while elsewhere in the world sial overlies the sima. The vertical discontinuity is near the andesite line, and in North America it is apparent when seismic surface waves of about 20 -sec. period and $70-\mathrm{km}$. wave-length cross from the Pacific Ocean bed to the land. There is much loss of energy. The discontinuity must extend at least some $25 \mathrm{~km}$. down, but its vertical extent does not exceed $200 \mathrm{~km}$. Such loss of energy is not apparent when similar waves cross other ocean beds, and if the evidence of seismic sounding is confirmed the North Atlantic depression has probably been caused by flexure and not by faulting.

Around the circum-Pacific line of instability in
Japan, the Philippines and California, the direction of faulting shows the land moving southerly with respect to the seaward side. Is this the Polfluchtkraft at work in the Pacific region?

The Atlantic and other regions would appear to be more doubtful. We must be careful, however, not to take all surface faults to be the cause of earthquakes. The cause of an earthquake and its focus may be more deep-seated, and the surface fault may be a result of the shock, not the cause of it.

Thus far we have not yet dealt with deep-focus earthquakes, that is, those with foci between 300 and $700 \mathrm{~km}$. deep. The work of Scrase (recognition of $p P$, etc.), Turner (waves at the antipodes), and Stoneley and Jeffreys (small amplitude $L$ and $M$ ) show that these undoubtedly occur. The Brunner depth chart constitutes a quick working tool for finding such depths. It appears that these deepfocus earthquakes could be caused by displacements resulting from the action of convection currents. The experiments of $\mathrm{H}$. Bénard in 1900 showed the presence of convective 'cells' in fluid between two parallel surfaces maintained at different temperatures. Pekeris has continued this work. Deep-seated heat from the still molten core of the earth some $3,500 \mathrm{~km}$. in radius may give rise to discontinuous geologically slow convection currents from this depth to, say, the $700 \mathrm{~km}$. discontinuity. This discontinuity may be between vitreous and crystalline basic material and is probably marked by a magnetic discontinuity also. Below $700 \mathrm{~km}$., forces would be dissipated by plastic flow, but just above $700 \mathrm{~km}$. the forees might accumulate until rupture, causing an earthquake, could occur.

The part played by radioactive heat at these depths is uncertain, though heterogeneities in the interior are more important than homogeneities in deciding where convexion currents shall be, and where the elastic potential shall accumulate. Western South America, the Sunda Sea near Java, and the region between Japan and New Zealand are very active regions at the moment. A series of observations of the direction of faulting taken over large areas of the earth's surface, also the careful integration of seismograms to discover the initial movement at the focus of deep-focus earthquakes would perhaps assist in finding the direction of deep-seated convection currents should these in reality exist.

1 Fiftieth Anniversary vol., Geol. Soc. Amer., June, 1941.

Trans. Amer. Geophys, Union (1941).

${ }^{3}$ Nature, Feb. 11, 1939.

NATURE, Tan. 6. 1940.

\section{RAMAN'S THEORY OF SPECIFIC HEAT OF CRYSTALS}

\author{
By DR. W. H. GEORGE \\ Chelsea Polytechnic, London
}

$\mathrm{W}$

HEN, in 1907, Einstein published his work on the specific heat of solids, nothing was known of X-ray crystallography. Crystals were apt to be regarded as a highly specialized form of solid matter. Now, as a result of the wide application of X-ray analysis to all solids, the crystalline state is known to be the normal stable state of solids. Theoretically, the crystal is a periodic three-dimensional grouping in space of an immense number of atoms, ions or molecules held together by interatomic or intermolecular forces. The unit cell, the smallest portion 
of the crystal to contain all the groupings of atoms characteristic of that particular form of the substance, is very much smaller than the smallest visible crystal. Nevertheless, the strength of diamond and the greasiness of graphite are inherent in the two different patternings of carbon atoms in the two different unit cells.

Properties of solids may be divided into the macroscopic, modified greatly by the shape and size of the individual specimen, and the sub-microscopic, inherent more in the patterning within the unit cell. This division of the physical properties of solids raises crucial questions when thermal properties are considered. On the kinetic theory of heat, heat is ' $a$ mode of motion'. Are the thermal vibrations of atoms in a solid characteristic of the unit cell, or are they dependent more upon the size and shape of the particular crystal, differing for different crystals of the same substance? Sir C. V. Raman discusses these problems in a summary of his work published with six experimental studies on the subject in the Proceedings of the Indian Academy of Sciences (A, 14, November, 1941). The following is an account of the theory and of Raman's criticisms.

\section{Raman's Specific Heat Theory}

Let it be supposed that the thermal vibrations of a crystal may be described without reference to the fine structure of the solid. We shall be dealing with elastic vibrations. Their modes and frequencies will depend upon the speed of propagation of elastic waves and upon the dimensions and boundary conditions of the crystal. Oscillatory motions of large groups of atoms will be involved, and the larger the crystal the smaller would be the frequency differences between the successive possible modes of vibration. In the limit, the elastic vibrations of a visible crystal would give a continuous spectrum of frequencies. When, however, the wave-length of these vibrations becomes comparable with the size of the unit cell, the possibilities of another type of vibration must be considered. Here we should be concerned primarily with vibrations of atoms about their mean positions in the unit cell. The dimensions of the crystal would have a negligible influence and the frequencies of the vibrations would be much greater. Although the principal modes of vibration would be the same for all unit cells, larger-scale oscillations might occur in which there is a repetition in cells of a superlattice, the spacings being an integral multiple of the unit cell. All such vibrations would be monochromatic and would give approximately a continuous spectrum only if the number of types of superlattice was very great. In general, the first type of vibration would tend to give a continuous spectrum and the second a line spectrum in the infra-red region of the spectrum.

The distribution of thermal energy between the two types of vibration is treated as follows. The largest part of the thermal energy is taken to be that associated with the unit cell. If there are $p$ atoms in the cell, there will be $(3 p-3)$ modes of internal vibration and 3 translations for the cell as a whole. If $p$ be fairly large, the $(3 p-3)$ degrees of freedom appearing as vibrations would greatly exceed in number the 3 degrees of translation. Considering the superlattices and taking one in which the cells have twice the edge-length of the lattice cells first chosen, we have $(24 p-3)$ modes of internal vibration of the atoms and 3 translations. The former $(3 p-3)$ modes of vibration will be included, as well as others approaching them in character. In addition, new modes of vibration previously reckoned as simple translations of the unit cell would occur. Unless $p$ is very small, not many superlattice vibrations need be considered. The number of vibrations of the elastic type bears to the total number of degrees of atomic freedom a ratio of the same order of magnitude as the ratio of the volume of an individual atom to the cube of the limiting wave-length. When, therefore, the wave-length limit is even moderately large in relation to the lattice spacings of the crystal, the energy of such vibrations is a negligible fraction of the whole.

\section{The Debye Specific Heat Theory}

The specific heat theory of Debye is criticized by Raman on the grounds that it associates the thermal energy of the crystal with that of elastic vibrations. Experimentally it is found that elastic vibrations travel with high speed, whereas heat energy travels very slowly indeed, at diffusion rates. The limits of using a discontinuous structure as an approximation to a continuous structure or vice versa are illustrated by the well-known example of the modes of vibration of a periodically loaded string and of a uniform string. While the specific heat curve can be uniquely derived from the vibration spectrum, a knowledge of the specific heat data is insufficient for a determination of the vibration spectrum. The Debye formula with an appropriately chosen limiting frequency fits the specific heat data in certain cases, but fails for several elements crystallizing in the cubic system. The Debye function is the integrated sum of a con. tinuous sequence of Einstein functions over a range of frequencies with weights proportional to their squares and a sharp cut-off at an upper limit. Experimental data which fit a Debye function should therefore equally be capable of being represented as the sum of a finite number of Einstein functions with appropriate frequencies and weight factors.

In Einstein's earliest papers, he pointed out that approximate relations between atomic frequencies and elastic constants were to be expected from elementary considerations. No special significance can therefore be attached to the fact that elastic constants calculated from the specific heats come out of the right order of magnitude.

\section{Born's Theory of the Cyclic Lattice}

The Born theory is criticized on the grounds that in essence it is based on concepts derived from the macroscopic behaviour of elastic solids of the same kind as are used by Debye. Born's theory rests on his postulate of the 'cyclic lattice'. All the atomic displacements are taken to be periodic in a volume having the same shape as the unit cell of the lattice but of very great size in comparison with it. The postulate makes no distinction between the atomic vibrations of high frequency and the elastic vibrations of low frequency. All the possible vibrations are assumed to have wave-lengths which are submultiples of the external dimensions of the crystal. This is in contrast with the Raman theory, where the unit cell or integral multiples of it are taken as determining the characteristic vibrations. The size of crystal would affect the modes of vibration only in so far as the unit cells at the surface of a crystal differ from those in the interior. The differences 
would be of the order of the difference of specific heat as determined with a single crystal and with the same mass of small crystals.

\section{Spectroscopic Data}

Whereas the Debye and Born theories lead to vibration frequencies of a crystalline solid giving a continuous spectrum, the Raman theory leads to a line spectrum. The relevant experimental data may be got from studies of the Raman spectra, the luminescence of crystals excited by light or by electric bombardment, and the absorption spectra.

A striking feature of the spectrum of the radiation scattered when a monochromatic beam traverses a transparent crystal is the sharpness of the lines of displaced frequency appearing in the infra-red. This feature is characteristic of the lines associated with either extensional or distortional vibrations of the ions or molecules in the crystal lattice or with translational or rotational motions. The latter type fails to appear when the substance is in the amorphous or fluid state, being then replaced by a diffuse continuum. The monochromatism of the vibrations is therefore closely associated with the crystalline state. Two plates are reproduced showing this monochromatism associated with superlattice frequencies for quartz and diamond.

Detailed studies of the relevant data have been made in the. Physics Department of the Indian Institute of Science, Bangalore, and are presented in a series of six papers. R. Norris deals with white phosphorus and quartz, B. Dayal with the cubic metals (lithium, tungsten, gold, silicon and grey tin) and with the hexagonal metals (magnesium, zinc and cadmium), V. B. Anand deals with diamond and C. S. Venkateswaran with alkali halides. Good or improved agreement of theory with experiment is found by using the Raman theory instead of the Debye theory. Numerical comparisons using the Born theory appear to be missing. The bulk of the thermal energy is associated with unit-cell frequencies appearing as monochromatic lines in the infra-red. The residue, associated with superlattice frequencies, appears in the remote infra-red, is monochromatic and is relatively important only at low temperatures.

\section{ADVANCE IN INVENTION: ITS RELATION TO WORLD PEACE}

\section{BY D. CARADOG JONES \\ University of Liverpool}

A YEAR after the outbreak of the present War the results were published of a study of the causes of the failure to preserve peace. The members of the study group are described as mainly officers of international organizations with headquarters at Geneva, meeting unofficially and able therefore to contribute to the discussion without reserve. The publication was issued under the authority of the Carnegie Endowment for International Peace.

\section{World Unity}

The factors that led to war are classed under three heads : political, economic, spiritual. But all three, when critically examined, are revealed as a failure to visualize the world as an organic whole. "The most startling example of the collective failure" to take a world view, under the political head, they say "was the attitude adopted toward Germany, not only in the clauses of the settlement, but still more in the manner of their presentation. . . . In particular, the refusal to negotiate with Germany created an attitude of mind in that country that was never subsequently changed." Put the rulers of Germany under restraint ; yes. Make the German people suffer; impossible, unless we are prepared ourselves to suffer with them. However much they may appear to our limited vision to deserve punishment, by placing them in the dock we shall but bring judgment upon our own heads. We are reminded of an illuminating analogy due to that far-sighted statesman, Señor S. de Madariaga: "A nation, a limb of the world, misbehaves, i.e., it acts against the world community, which is the whole body. And sanctions are decided on to punish it. But, can we punish a limb without punishing the whole body ?" If we sow the wind, we reap the whirlwind.

The fundamental character of this principle of world unity comes out nowhere so clearly as in the economic aspect of international relations. The Geneva group recall the sequence of events following the attempt to extract huge reparations from Germany. Failure to grasp the fact that payment in goods would immediately put large numbers of people in the allied countries out of work may be taken as evidence that the mismanagement of affairs after the War of 1914-18 was due in large measure to a lack of understanding of elementary economics. It was as much a failure of the head as of the heart. Happily there are signs of greater enlightenment among those who are at present responsible for advising the leaders of the democratic Governments in such matters. In particular, they are fully aware of the consequences of the remarkable progress achieved in technological invention. This was causing so great a revolution in industrial production, even before 1914 , that it was bound sooner or later to lead to trouble between nations if each attempted to pursue its own selfinterested path regardless of the welfare of others; and this new industrial revolution may cause still greater disaster after the present War if its implications are not more widely and clearly realized. It is essential that Governments should be able to depend upon the support of public opinion for any concerted measures they may adopt for the control of output and its distribution, in the interests of the world as a whole and especially of the weaker nations.

\section{Progress in Output}

A few facts may be given which bear striking witness to the extent and the rate of advance in the output capacity of modern machinery. Colin Clark has made a comparison of the real income produced per head of the working population in different countries on the basis of a 48-hour week. His figures indicate a rise in Sweden and Japan to a level more than four times as high as that reached seventy or eighty years ago. In most highly industrialized countries the income so calculated has been at least doubled during the same period of years, and this was accomplished in spite of a considerable increase in population and decrease in the hours of work. In the last two decades the rate of progress has been even more astonishing. According to an estimate published by the Royal Economic Society, the increase in physical output per operative in the United 